Revista Herencia, Vol. 31 (2), julio-diciembre, 2018.

\title{
UN VIAJE A LA NIÑEZ COSTARRICENSE: TRIQUITRAQUE, REVISTA INFANTIL (1936-1947)*
}

A trip to costarrican childhood: Triquitraque, children's magazine (1936-1947)

\author{
José David Ramírez Roldán \\ Universidad de Costa Rica, Costa Rica \\ jrd_r@ghotmail.com
}

Recibido: $15-11-2018$

Aprobado: 30-11-2018

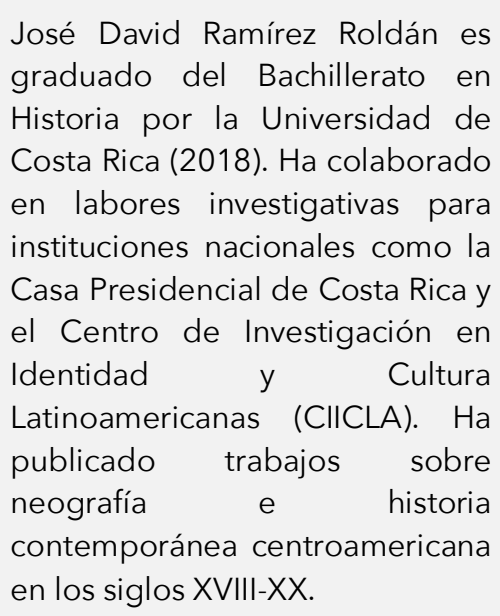

José David Ramírez Roldán es graduado del Bachillerato en Historia por la Universidad de Costa Rica (2018). Ha colaborado en labores investigativas para instituciones nacionales como la Casa Presidencial de Costa Rica y el Centro de Investigación en Identidad y Cultura Latinoamericanas (CIICLA). Ha publicado trabajos sobre neografía e historia contemporánea centroamericana en los siglos XVIII-XX.

\section{RESUMEN}

En términos generales, esta investigación ofrece hallazgos sistemáticos para todo el periodo de publicación sobre la revista infantil Triquitraque (1936-1947), la cual pasó a ser parte del acervo intelectual de la población infantil alfabetizada y escolarizada en Costa Rica durante la primera mitad del siglo XX. Sin dejar de lado que los escolares son transmisores de conocimiento en casa hacia sus padres o tutores, dicha publicación tuvo un planteamiento lúdico-educativo caracterizado gracias al conocimiento literario de sus codirectores en los contenidos, convirtiéndose en un órgano de difusión cultural nacional e internacional.

Palabras clave: Triquitraque; literatura infantil; escolarización; comunismo; publicidad

\begin{abstract}
In general terms, this research offers systematic findings for the entire period of publication about the children's magazine Triquitraque (1936-1947), which became part of the intellectual heritage of the literate and schooled children population in Costa Rica during the first half of the twentieth century. However, this work takes into account that schoolchildren are transmitters of knowledge at home to their parents or guardians, this publication had a ludic-educational approach characterized by the literary knowledge of its co-directors in the content, becoming a national and international cultural dissemination magazine.
\end{abstract}

Keywords: Triquitraque; children's literature; schooling; communism; advertising

\footnotetext{
*Este artículo es una versión resumida del trabajo final en Historia para obtener el grado de Bachiller. Veáse en: José David Ramírez Roldán. “Triquitraque, revista infantil (1936-1947)”. (Trabajo de graduación en Bachiller de Historia. Universidad de Costa Rica. San José, 2017). Inédito.
} 
Para mi abuela, padres y hermanos: Xinia, Rose Marie, Guillermo, Valeria, Evelyn y Fabian. A mis amigos y colegas: Josué y Jennifer. En especial al profesor Iván Molina, quien sin sus consejos, apoyo y orientación adecuada este trabajo jamás hubiera visto la luz.

\section{Introducción}

La noción de prensa infantil consiste en una serie de publicaciones periódicas destinadas al consumo de la niñez, conformada por formatos de diarios, revistas o historietas (cómics). Se puede afirmar que históricamente la prensa infantil se enmarca en esa nueva concepción de la niñez, desde su surgimiento europeo y latinoamericano a fines del siglo XVIII, sin embargo, para el caso costarricense aparecen a inicios del siglo XX. Asimismo, se puede caracterizar específicamente por su cuidada impresión, alto contenido ilustrativo, no muy extensa. Por lo general, en sus inicios son de distribución por suscripción y tienen un fin pedagógico de crear conocimiento general, y no siempre con precios cómodos para la época.

En Costa Rica, después de la primera revista exitosa para niños, llamada San Selerín (1912 a 1913 y 1923 a 1924), apareció para el año de 1936 la publicación de Triquitraque: revista infantil en el escenario literario nacional, convirtiéndose en la obra infantil de este tipo más longeva en la primera mitad del siglo XX, dejándose de publicar hasta 1947, con una pequeña interrupción durante 1943. Así, este impreso logró superar en duración a su antecesora, siendo producida en su primer año por la Asociación de Maestras de Kindergarten y posteriormente codirigida por las figuras literatas de Carlos Sáenz, Luisa González, Lilia Ramos y Adela Ferreto, todos docentes formados en la Escuela Normal de Heredia, además de estar afiliados como militantes intelectuales al Partido Comunista de Costa Rica (P.C.C.R.).

Este es un trabajo que presenta los hallazgos sistematizados de todo el periodo de publicación de la revista infantil Triquitraque, la cual pasó a ser parte del acervo intelectual de la población infantil alfabetizada y escolarizada en Costa Rica durante la primera mitad del siglo XX. Dicha publicación nacía con un planteamiento educativo que finalmente se convirtió en un proyecto cultural complejo para la época. De hecho, el objetivo de esta investigación es estudiar el papel de la revista costarricense para niños Triquitraque en la sociedad costarricense durante la primera mitad del siglo XX, siendo un objeto de estudio 
viable, innovador y tratable desde el contenido de las publicaciones que no han sido explotadas con anterioridad de forma sistemática y que estén vinculas a la historia de la producción literaria de los comunistas en Costa Rica, pero en este caso, de tipo infantil.

Como se puede apreciar por medio de las fuentes primarias (sus publicaciones), este trabajo retoma las condiciones históricas del país para publicar una revista infantil tan longeva y el ambiente intelectual-educativo en que Triquitraque estaba inmersa durante su delimitación temporal, tanto nacional como internacional, o sea, dentro de sus años de publicación, mayo de 1936 hasta octubre de 1947, exceptuando un análisis profundo para su respectiva interrupción durante 1943, dejando un total de 91 números publicados en toda su historia. Por su lado, esta investigación presenta algunas limitaciones y posibilidades para estudiar sus diversos aspectos de producción con precisión, es en este punto donde se evidencia los pros y contras de este trabajo, dichas se pueden apreciar de la siguiente manera: la falta del registro contable de lo publicado, el número exacto de suscriptores de manera progresiva, entre otros aspectos que son difíciles de dar con pistas, más que todo a lo que se refiere a su producción material. Sin embargo, sí se pueden observar algunas cifras de la magnitud e importancia de Triquitraque en el país, que son imposibles de no manejar para realizar el presente estudio, como: la larga duración de la revista (10 años), el número promedio de páginas (16 en tamaño tabloide), su continua periodicidad (una vez al mes). Todo lo anterior indica que la revista no dejó documentación de apoyo para rellenar algunas lagunas históricas de forma concreta, o al menos se desconoce de su existencia. Empero, por medio de los fragmentos o vestigios indirectos se pueden inferir algunos datos de la producción de la revista con los cuales podemos confrontar los problemas de investigación, aunque no con total exactitud.

La delimitación espacial trata de dar cuenta de la distribución de la revista en el territorio nacional (Costa Rica) y otros territorios extranjeros de manera segundaria en donde hubo intentos por distribuirse de forma continua, como: Venezuela, Panamá, Centroamérica y México. Pero en una situación donde las escuelas del Valle Central parecen tener la mayor difusión de la obra debido a las condiciones del contexto, ya que existía una fuerte relación no sólo intelectual, sino que también productiva con la Asociación de Maestras de Kindergarten para su debida publicación periódica, provocando una concentración dirigida hacia la población letrada en el país de aquellos años. 
Por otro lado, en términos más específico, los propósitos de esta investigación serian analizar el surgimiento y desarrollo de la revista infantil Triquitraque para comprender las estrategias de difusión por parte de la codirección; identificar los autores que colaboraron en el contenido del impreso con el fin de rastrear las comunidades intelectuales que se establecen alrededor de la obra; y estudiar cómo se presentaban las diversas temáticas y contenidos tratados en la revista para entender cómo se dio el desarrollo de su impresión al obtener un éxito temprano y longevo en el campo de los impresos infantiles. Las fuentes utilizadas para este trabajo consisten en un tratamiento para el análisis de contenido (en su mayoría literario y en menor medida publicitario) para todos los números publicados por la revista Triquitraque que fueron localizados. Cabe señalar que, en su mayoría, los números estudiados del impreso fueron recopilados casi en su totalidad por medio del acceso digital a la Biblioteca Nacional de Costa Rica. Empero, con esta obra se pretendió utilizar sus 11 años totales de publicación desde 1936 hasta 1947, con una evidente interrupción durante 1943 y algunas faltas para reconstruir toda la producción. Como ventaja de las fuentes utilizadas en este trabajo se debe decir que todos los números publicados traen completamente la información para extraer todos los indicadores de la investigación.

La estrategia metodológica consiste en historizar la trayectoria de la revista Triquitraque se utiliza un análisis cualitativo de sus contenidos generales en donde se incluirá en la base de datos para su respectivo desmenuzamiento de datos claves. Para ello, los contenidos están organizados con un tratamiento de la información recolectada de forma separada temáticamente para evitar entremezclar dos puntos neurálgicos, pero disimiles entre sí para este trabajo. Así, se extrae desde una base de datos que abarca específicamente los contenidos generales y literarios del impreso las siguientes características: fecha de publicación del número, el nombre de la imprenta, el precio de venta (en colones), su autor, año de publicación, número de publicación, el sexo y la nacionalidad del autor, el título del texto publicado, el tipo del texto que se publica en el impreso, específicamente, los poemas con su respectiva temática, la paginas en que aparecen la aparición del trabajo de los autores, hasta nombrar a los encargados de la dirección y administración en diversos momentos históricos de la revista. Además, se cuenta con otra base de datos de anuncios publicitarios, a favor de sistematizar la cantidad de empresas que se publicitaban en todos los años de publicación de la revista según la provincia de Costa Rica, esto con el fin de comprender el valor comercial del insumo y la proveniencia de sus finanzas privadas. 


\section{Surgimiento y desarrollo de Triquitraque}

1. El contexto de la revista en la Costa Rica de los treinta y cuarenta

En los años treinta en Costa Rica las clases subalternas carecían de una organización política que orientara sus exigencias sociales, en un momento de coyuntura frente a la lucha sindical que iba en incremento debido a una crisis económica mundial a inicios de 1930. Ante ese panorama, nació el denominado Partido Comunista de Costa Rica en el año 1931, el cual logró retomar los sectores históricos que estaban en las luchas sociales, como: organizaciones laborales, sindicatos, mutualistas, educadores e intelectuales ${ }^{1}$.

En este contexto la orientación de izquierda llegó a construir toda una problemática de la cuestión social desde los medios de difusión cultural (especialmente, impresos culturales) durante los años treinta y cuarenta. Debido a este factor llegaron a tener un gran desempeño como fuerza política, a pesar de ser un partido de reciente formación, participando en las elecciones a nivel nacional de 1936 con el nombre Bloque de Obrero y Campesinos, así en el año 1936 el ganador de las elecciones presidenciales fue el anticomunista y simpatizante de ideas fascistas, León Cortés, siendo candidato por el Partido Republicano Nacional ${ }^{2}$.

En estas dos décadas, los comunistas se forjaron como fuerza social por medio de la difusión de sus ideales gracias a la elaboración de su propia política intelectual, con su periódico Trabajo, dicho impreso logró colocarse en la política nacional de la sociedad costarricense en aquellos años desde una cultura comunista apelando a la identidad de carácter obrero y costarricense ${ }^{3}$. Este dato anterior revela la importancia para entender la preparación, calidad, contenido y experiencia que poseía la revista infantil Triquitraque al ser dirigido por militantes y simpatizantes a las ideas del Partido Comunista de Costa Rica, lo cuales entendían la función política, económica, cultural y social en el mercado de los impresos culturales. Para asegurar la actividad inicial de la

\footnotetext{
${ }^{1}$ Ana María Botey. La fundación del partido comunista de Costa Rica, 1931. (Tesis para optar por el grado de licenciatura en historia. San José: Universidad de Costa Rica, 1981), pp. 50-51.

${ }^{2}$ Ana María Botey, Costa Rica entre guerras: 1914-1940. (San José: EUCR, 2005), pp. 100-101.

${ }^{3}$ Botey Ana María, "Las representaciones sociales de la pobreza en la Costa Rica de la década de 1930". En: Viales Hurtado, Ronny José (comp.). (Pobreza e historia en Costa Rica: determinantes estructurales y representaciones sociales del siglo XVII a 1950. San José: EUCR, 2005), p. 279.
} 
política del Partido Comunista de Costa Rica fue fundamental la llegada al poder presidencial de Ricardo Jiménez (1932-1936), consolidando su legalidad como organización durante la década de los treinta sin ser perseguidos o detenidos durante la administración de León Cortés (1936-1947), ya que los comunistas estaban inmersos en el marco legal e institucional del país que había sido por fin consolidado para no permitir su ilegalización ${ }^{4}$. Sin embargo, eso no quiere decir que la contienda entre Estado y los comunistas no haya sido problemática en la mayor parte de la historia nacional.

Así, la revista infantil Triquitraque se llega a publicar hasta junio de 1936, o sea, en un contexto de represión anticomunista e inclinaciones fascistas reciente por parte del gobierno, ya que la administración de Cortés fue reconocida por su persecución a los opositores principales, los denominados comunistas, reconocidos como enemigos políticos. Fue tal el nivel de anticomunismo de su presidencia que llegó a desconocer el resultado electoral emitido por el Gran Consejo Electoral durando el medio periodo de 1938. Finalmente, eligió a un allegado de su confianza, anulando la escogencia del director de Triquitraque, Carlos Luis Sáenz, el cual había sido elegido por la provincia de Heredia. Además, durante aquel mandato se destituyeron de sus puestos algunos maestros que eran opositores al régimen, muchos de ellos con orientación comunista ${ }^{5}$.

Triquitraque no fue utilizado por sus coeditores para ser contestatario de manera frontal contra la administración de Cortés de forma directa, posiblemente por miedo a un despido o al corte de los fondos públicos que los financiaban, pero sí de manera general realizaron y organizaron criticas mordaces al fascismo que había en el país durante aquellos años debido a la aprobación presidencial de ese tipo de ideas, y más a fines de los años treinta con la llegada al poder en Alemania del Partido Nacional Socialista, también en las páginas del impreso se propició los regionalismos de manera educativa para fortalecer otra visión de identidad nacional más variadab, lo cual era una noción del país más multicultural, elemento de construcción identitario alternativo y contestatario para aquel contexto reaccionario.

Al entrar en la década de los años cuarenta, el Partido Republicano Nacional continua en el poder, precisamente para disputarle el voto popular a los

\footnotetext{
${ }^{4}$ Iván Molina, "Ricardo Jiménez y los comunistas en la Costa Rica de la década de 1930". Inter.c.a.mbio, Año 4, N. 5 (2007), pp. 95-96.

${ }^{5}$ Botey, "Las representaciones sociales de la pobreza...", 103.

${ }^{6}$ Ramírez Roldán José David, 2017. "Base de Datos: Triquitraque: contenidos generales. 1936-1947".
} 
comunistas, inició todo un programa de reforma social apoyado por la lglesia católica, posteriormente, en la suma, dicho proyecto se convertiría en la atracción para dar una alianza con los comunistas, logrando que ya no se combatieran entre sí más estas dos fuerzas políticas. El Republicano Nacional puso su apoyo como candidato presidencial al Dr. Rafael Ángel Calderón Guardia para las elecciones de 1940, lo cual se dio nuevamente con un triunfo exitoso para que la ya consolidada institución llegara a poseer indiscutiblemente la mayoría del apoyo nacional en términos cuantitativos de votos ${ }^{7}$. Sin embargo, cuando Teodoro Picado Michalski llega al llegar al poder presidencial (19441948) empieza su administración a romper las alianzas con el comunismo en los primeros años de su administración, en desgracia de los puestos laborales de los militantes de izquierda ${ }^{8}$. Así, se podría decir que en los años cuarenta la administración de Calderón Guardia (1940-1944) hasta el cambio presidencial de Picado Michalski (1944-1948) se desarrollaron en un contexto que estuvo sometido a encuentros y desencuentros políticos en cuanto a las tenciones con los intelectuales de izquierda del país se refiere.

Asimismo, por su lado, Monseñor Sanabria como representante de la Iglesia católica contribuyó con su respectiva bendición a los propósitos del gobernante, dentro del marco de una reforma social. De dicha manera, los intereses de los comunistas, con los del gobierno y la Iglesia se unificaron por un momento con un fin reformista a favor de los sectores subalternos, volviendo a cambiar el nombre de la agrupación comunista en 1943 debido a sus alianzas políticas, denominándose, Partido Vanguardia Popular. Además, por un breve momento, debido al contexto internacional de la Segunda Guerra Mundial, no fue mal vista por las potencias mundiales la unión entre el capitalismo y el comunismo en contra de la amenaza del nacionalsocialismo, y posteriormente, sí se convirtió en un problema grande al fin del altercado bélico mundial dando inicio a la Guerra Fría (1945-1991) entre aquellos dos polos políticos irreconciliables. Por ende, las contradicciones empezaron a aparecer debido al acercamiento que estuvo mediatizado por las diferencias ideológicas del momento, pues convivían las luchas anticomunista por parte de la lglesia, hasta observarse como la alianza inició y finalizó fortuitamente debido al contexto internacional de guerra, lo que posteriormente, abriría pasó nuevamente al anticomunismo más recalcitrante y en el caso costarricense a una mayor actividad política por parte de los

\footnotetext{
${ }^{7}$ Molina, "Ricardo Jiménez y los comunistas...", 95-96.

${ }^{8}$ Gerardo Contreras, "Arnoldo Ferreto Segura y el Partido Comunista de Costa Rica en la lucha por la segunda y auténtica Independencia Nacional". Revista Estudios UCR, No. 22 (2009), p. 67.
} 
comunistas a favor de luchar por sus intereses políticos y los logros sociales conseguidos?.

Este contexto imposibilitaría más tarde la continua producción de Triquitraque en 1947, pues se embarcarían sus productores a dar una lucha política más intensa para mantener las reformas sociales en pie. Por ende, se podría decir que los comunistas fueron imposibilitados únicamente a seguir su trabajo durante la administración presidencial de Picado, puesto que el Partido Republicano Nacional ya para 1946 había dejado florecer una ruptura en la alianza con la izquierda política y empezaba a llenarse nuevamente aquel ambiente de guerra fría de anticomunismo ${ }^{10}$, las actuaciones de los militantes debían redireccionarse no a los medios educativos en ese contexto, sino a la política directamente.

A un lado a esto, Picado ordenó al señor Ministro de Educación Pública durante su mandato que se despidiera a varios educadores considerados comunistas y que ejercieran en el Magisterio Nacional, esto debido a que profesaban la ideología marxista-leninista, como en el caso de un familiar cercano de la nueva administradora de Triquitraque, pues Arnoldo Ferreto Segura quien era hermano de Adela Ferreto llegó a sufrir este tipo de persecución política. De alguna forma, Adela Ferreto era la nueva cara principal de dicha revista infantil, dicho despido de su pariente y otros comunistas, posiblemente se le cortó el financiamiento a la revista en 1947, pues al mandatario Picado veía pernicioso el trabajo cultural de la comunista Luisa González en Triquitraque, siendo otra opción que a raíz de todo la persecución política se diera el repentino cambio administrativo después de tantos años sin transformación alguna, pues durante su gobierno también se dio el final de la obra con la cual los infantes disfrutaban tanto en la Escuela Maternal y diversas escuelas del país ${ }^{11}$. Asimismo, otro dato sumamente importa es como el periódico comunista Trabajo desapareció al poco tiempo después, más precisamente en febrero de 1948, ya que la Guerra Civil empezaría el día 12 en el mes de marzo del mismo año ${ }^{12}$.

\footnotetext{
${ }^{9}$ Iván Molina y Fabrice Edouard Lehoucq. Urnas de lo inesperado. Fraude electoral y lucha política en Costa Rica (1901-1948) (San José: EUCR, 1999), pp. 155-161.

${ }^{10}$ Molina y Lehoucq, "Urnas de lo inesperado...", 181-182.

11 Contreras, "Arnoldo Ferreto Segura y el Partido Comunista...", 67.

${ }^{12}$ Iván Molina, "Los comunistas y la publicidad en Costa Rica. El caso del periódico Trabajo (1937-1948)". Secuencia. Revista de Historia y Ciencias Sociales, No. 77 (mayo- agosto, 2010), p. 67.
} 


\section{Codirectores}

Triquitraque es la revista cultural para niños de mayor duración y trascendencia en Costa Rica durante la primera mitad del siglo XX, y a pesar de eso, únicamente estuvo elaborada en su primer número por un grupo de maestras de preescolar y en las ediciones siguientes por cuatro personas destacadas de manera personalizada, siendo dirigida por los militantes comunistas Adela Ferreto y su esposo Carlos Luis Sáenz Elizondo, y administrada por Luisa González Gutiérrez y su hermana Lilia González Gutiérrez ${ }^{13}$. Dichos militantes comunistas y docentes escolares fueron producto de un contexto histórico relacionado a una formación educativa que los llevaron a tener una visión extensa de la cultura impresa, y en el caso de Triquitraque, por medio de un formato elaborada para niños.

Así, Triquitraque era un proyecto cultural de carácter conjunto, publicada por la Asociación de Maestras de Kindergarten ${ }^{14}$, por ende, sus métodos interactivos estaban influenciados por visiones pedagógicas de maternales experimentales. Lo anterior sucedió desde inicios de los años treinta gracias a las inclinaciones que profesaba la asociación al estar adjunta al Kinder Laboratorio de la Escuela Normal y la Escuela Maternal Montessoriana, las cuales fueron fundadas de la mano de maestras, activistas políticas de izquierda y escritoras infantiles como la misma Luisa González Gutiérrez, siendo centros para el desarrollo de educación preescolar pública con una orientación pedagógica experimental ${ }^{15}$.

Así, la visión escolar de Triquitraque tenía como fin ofrecer un debido desarrollo físico y social del niño a través de medios lúdico-educativos con fines interactivos, por eso la revista fue un medio más de este tipo de visión clara en términos de manera de proyectarse sociablemente, siendo entre la literatura infantil y la educación escolar exitosamente dirigida. Entonces, es posible afirmar que Triquitraque era un medio más de los movimientos populares de alfabetización y de promoción de la esfera educativa, siendo un recurso útil para cultivar las identidades nacionales hasta los símbolos y lealtades patrióticas de manera alterna por medio de otros formatos de estudio. Para aclarar la idea anterior, sería bueno señalar que la imagen de temáticas patrióticas fue únicamente creada por la misma revista y su codirector Carlos Luis Sáenz para

\footnotetext{
${ }^{13}$ Ramírez Roldán José David, 2017. “Base de Datos: Triquitraque: contenidos generales. 1936-1947”.

14 Ibid.

15 Margarita Dobles Rodríguez, Literatura infantil (San José: EUNED, 1981), p. 4; Ana Patricia Rodríguez. Dividing the Isthmus: Central American Transnational Histories, Literatures, and Cultures (USA: University of Texas Press, 2009), p. 57.
} 
fines de los años treinta y los años cuarenta del siglo $X X$, más que todo con el uso de textos históricos ${ }^{16}$ y poemas ${ }^{17}$ (pero dichos formatos serán tratados más adelante a profundidad, específicamente en el tercer parte).

Durante todo el primer año, simplemente la revista aparece con el nombre de Asociación de Maestras de Kindergarten, al igual que en los casos que no tienen referencia personalizada, y en el primer número de abril de 1947 se revela que la administradora de ese momento sería su hermana Lilia Gonzáles Gutiérrez ${ }^{18}$. Asimismo, llega aparecer de manera más profesional según la división del trabajo de la obra con el nombre de Carlos Luis Sáenz como director, este cargo empieza a compartirlo en 1947 con su esposa Adela Ferreto. Ahora bien, la revista fue codirigida mayoritariamente por los intelectuales costarricenses Carlos Luis Saénz, Luisa González y Adela Ferreto, todos escritores infantiles y maestros formados en la Escuela Normal de Heredia, además estos personajes eran todos militantes a las ideas del Partido Comunista de Costa Rica (P.C.C.R.). Lo anterior, es relevante para entender la inclinaciones y formaciones pedagógicas de Luisa González, la cual fue la principal administradora a lo largo de 1938 y 1939, como también durante los años 1941 y 1942 según lo referenciado en la revista como coeditora, posiblemente su participación productiva se dio en conjunto para otros números pero los números con estas excepciones aparecen sin regencias de administración y dirección personalizada, pues es en 1944 hasta octubre de 1946 que regresa nuevamente su nombre en un número doble (números $84-85$ ) del impreso ${ }^{19}$.

Por otro lado, la figura de Luisa Gonzáles Gutiérrez desde antes de la producción de Triquitraque aparecía en la esfera educativa como destacada escritora costarricense de origen herediano, graduada en la Escuela Normal de Costa Rica como maestra primaria a inicios de los años veinte. Empero, fue pionera de Maestros Unidos, una asociación que dio origen a la Asociación de Maestros Unidos $(A N D E)^{20}$, y cabe señalar que dicha institución estuvo bastante interesada en la producción educativa de Triquitraque.

De hecho, el periodo de intervención (1943) de la revista se aclara con causas justificadas hasta el año de 1944 debido a falta de fondo económicos, expresándose que su regreso fue únicamente posible gracias a que la Secretaría

\footnotetext{
16 Triquitraque: revista infantil, 1947-09-01: 8-10.

17 Triquitraque: revista infantil, 1939-06-01: 4.

18 Triquitraque: revista infantil, 1947-04-01, 7.

19 Ramírez Roldán José David, 2017. "Base de Datos: Triquitraque: contenidos generales. 1936-1947".

${ }^{20}$ Elías Zeledón Cartín, Biografías de costarricenses (Heredia: EUNA, 2013), pp. 97-98.
} 
de Educación que aprobó un apoyo económico solicitado por la Asociación Nacional de Educadores (ANDE), según lo expresado en una carta de agradecimiento por un profesor hacia Luisa Gonzáles con respecto a la continuación del impreso. Empero, en esa presentación de la revista también se señaló a la anterior revista infantil San Selerín como un proyecto predecesor ya muerto que se había dado en el pasado, utilizando esos recursos nostálgicos para alimentar la continuidad de apoyo para Triquitraque. En ese mismo número, se deja en claro que para no aumentar el precio de la revista (10 centavos de colón ${ }^{21}$ ), la ANDE en 1943 tuvo que pedir la asistencia económica de la Secretaria de Educación, encabezado por Luis Demetrio Tinoco, y que llegó a solucionarse hasta al año siguiente por medio de Hernán Zamora ${ }^{22}$.

No es imposible entender el proceso pedagógico en Costa Rica durante la segunda mitad del siglo XX en toda su complejidad, menos si no se toman recursos como Triquitraque para dar cuenta de la memoria nacional en términos de un proceso histórico de carácter educativo y cultural que se dio en Costa Rica de la mano de ideas que provenían de la izquierda política, y menos sin tener en cuenta la figura femenina de Luisa y Lilia Gonzáles o la misma Adela Ferreto, siendo esta revista infantil pionera como una herramienta pedagógica útil y que contaba con suma popularidad en el país. En resumen, la revista tiene que verse como un recurso que representaba una gran parte de la colaboración de los maestros y literatos al sistema educativo del país que recaía en el contenido literario del impreso, material que en su mayoría poseía tendencias a simpatizar con el fortalecimiento a la literatura nacional e internacional, pero sin dejar de lado el valor pedagógico de la misma, revelando el esfuerzo de producir una revista del calibre de Triquitraque de sus coeditores.

Cabe señalar, que la participación especifica de las hermanas Gonzáles (Luisa y Lilia) en la revista se destinó en la obra prácticamente para su administración, pero nunca en el papel de directoras, y es prácticamente nula la aparición de estas intelectuales en colaborar directamente como escritoras en algún formato literario de la revista, o por lo menos, sus nombres no aparecen explícitamente y no es posible concluir que hayan ocupado algún seudónimo o anónimo ${ }^{23}$.

En el caso de Adela Ferreto, sí se destaca quince veces su participación como escritora en la revista, y con una participación de suma relevancia en el último

${ }^{21}$ Cabe señalar que este valor de venta se mantuvo en todos los diez años de producción de la revista.

22 Triquitraque: revista infantil, 1944-04-01: 2.

${ }^{23}$ Ramírez Roldán José David, 2017. "Base de Datos: Triquitraque: contenidos generales. 1936-1947". 
año de vida del impreso ${ }^{24}$. Así, para el mes de mayo de 1947, un cuento de su autoría aparece siendo contando con excelentes ilustraciones dibujadas de casas creadas con diversos materiales autóctonos como madera, adobe, paja y ladrillo, llegando acaparar hasta tres páginas de la revista ${ }^{25}$, siendo relevante su participación por un breve instante. Sin embargo, su participación en la dirección llega hasta junio de 1947, pero su aparición desde mayo de 1944 hasta mayo de 1947 como escritora de cuentos, textos históricos y poemas ${ }^{26}$, pocas veces referida directamente con su nombre completo de soltera, y la mayoría de las veces con el seudónimo de A de Sáenz, o sea, su nombre de casada Adela de Sáez, haciendo alusión a su matrimonio con Carlos Luis Sáenz ${ }^{27}$.

Ahora bien, el único hombre que era codirector de Triquitraque, fue el destacado escritor costarricense, oriundo de Heredia, Carlos Luis Sáenz Elizondo, teniendo en común espacios políticos y literarios con sus similares, además de un alma mater como la Escuela Normal al igual que su esposa Adela Ferreto (Adela Sáenz) y sus compañeras de trabajo ${ }^{28}$. Sáenz no solo destacó como maestro tempranamente, sino fue quien más contribuyó en Triquitraque por medio de diversos formatos literarios y dio mano destacadas veces en las presentaciones del impreso, esto volviéndolo atractivo por lo uniforme de la escritura para el ojo del lector. Destacadas veces, la autoría de la presentación y otros formatos literarios presentados en la revista eran revelados explícitamente con su nombre por completo como autor o se sobreentendía su autoría con pseudónimos como C.L.S. o Gulliver, Lemuel, así algunos de estos primeros casos se dan en julio de 1936, cuando aparece el tema de los transportes aéreos y los aviadores para introducir el impreso, apareciendo los personajes de Tío Conejo y Triquitraque como viajeros de un avión ${ }^{29}$, esto a manera de verso, desecándose la habilidad de Sáenz en su participación de volver atractivo el impreso con figuras literarias y temas populares. La explotación literaria de Sáenz llama la atención, pues llega a ser uno de los escritores más destacados en la revista como poeta, dramaturgo y cuentista, sin dejar de lado su participación con artículos informativos en temas educativos de corte patriótico como la independencia ${ }^{30}$.

\footnotetext{
24 Ibid.

${ }^{25}$ Triquitraque: revista infantil, 1947-05-01: 5-7.

${ }^{26}$ Ramírez Roldán José David, 2017. "Base de Datos: Triquitraque: contenidos generales. 1936-1947”.

${ }^{27}$ Triquitraque: revista infantil, 1947-05-01: 5-7.

${ }^{28}$ Zeledón, "Biografías de costarricenses", 97-98.

29 Triquitraque: revista infantil, 1936-07-01: 4.

30 Triquitraque: revista infantil, 1947-09-01.
} 
Cabe señalar, que hasta horóscopos llegó a lanzar la revista de la mano de Sáenz, los poemas infantiles eran su punto fuerte pues era en lo que más se había especializado en la revista, ensalzando con su prosa hasta el día de la madre $^{31}$ y la figura enaltecida de Juan Santamaría ${ }^{32}$; posiblemente otras presentaciones de la obra también corrieron a su cuenta, pero de manera anónima. Lo anterior deja claro el papel literario y no solo directivo que tuvo Carlos Luis Sáenz en la obra, en diversas aristas de la literatura o arreglos en otros cuentos tradicionales conocidos y hasta mezclando todos esos insumos durante la administración también. Con ello, es posible dejar en claro lo multifuncional y diligente del trabajo de sus coeditores, utilizando recursos como seudónimos e introduciendo temas de manera continua número tras número con cierta lógica temática, sin dejar de lado de su participación en diversos formatos literarios.

\section{Estrategias de financiamiento}

Las estrategias de financiamiento rescataban toda una maquinaria interesante para el éxito longevo de la revista en comparación con la corta vida de los impresos similares anteriores, ya que parte de las estrategias de financiamiento empleadas por Triquitraque para administrar su producción, durante prácticamente toda su existencia, si exceptuamos las entradas posteriores de la amplia publicidad en sus páginas que llegó a tener desde el segundo año de publicación, podemos argumentar que se basó en una gestión no comercial, que consistía en responsabilizar a los niños como corredores (agentes) de su distribución o venta, para buscarle nuevos compradores y/o suscriptores del impreso al por mayor.

Sin embargo, también el apoyo de las contribuciones de donaciones monetarias podía tener relevancia para su elaboración. Empero, hay que tener en claro que la financiación esa con fines difusivos y no lucrativos, como en aquel marzo de 1941, cuando el impreso deja en claro a los clientes que cancelen sus cuentas atrasadas, y sino la revista se iba a atrasar, puesto que "...la revista no es comercial, sino se preocupa por la cultura infantil costarricense" ${ }^{\prime 33}$.

Sin embargo, lo más importante recae en su valor de subscripción anual (0.80 colones), pues era notable como constantemente por medio de avisos de

\footnotetext{
31 Triquitraque: revista infantil, 1937-08-01: 3.

32 Triquitraque: revista infantil, 1946-03-01: 15.

33 Triquitraque: revista infantil, 1941-03-01: 15.
} 
morosidad se le pedía cancelar a los usuarios con la revista sus deudas respectivas, advirtiendo que era posible la detención de seguir produciéndose si no se hacia el pago a tiempo. También hay que tener en cuenta que esto era un arma de doble filo al haber cuentas que nunca se pudieron cobrar (contablemente encobrarles). Asimismo, otro método que volvió atractiva a la revista y ayudó a su difusión recae en la posibilidad de que los niños colaboren como corresponsales y/o autores de escritos, sin dejarlos únicamente como vendedores, siendo una vía más para obtener ganancias personales.

En término de estrategias de financiamiento existieron recursos muy sobresalientes en los tantos números de la revista Triquitraque, destacándose las felicitaciones a sus lectores, este elemento logra atraer a los niños de diversas maneras, desde una vía personalizada por sus aportes intelectuales a la revista, pasando por agradecer a diversos grupos o escuelas por los trabajos realizados durante su vida escolar. Es en este punto donde se denotan el alcance difusivo del impreso en las provincias del país como Heredia, San José, Puntarenas, Alajuela, Cartago (destacándose hasta el periférico pueblo de Tierra Blanca) ${ }^{34}$. Así, la revista consiguió llegar a una enorme popularidad al alcanzar los 10.000 lectores en la edición de $1945^{35}$.

También, existía por lo general una sección de concursos, dotada de premios como juguetes, libros o material didáctico, de hecho, fue tan grande el éxito de esta estrategia difusiva que la revista direccionaba de sus financias con hasta 25 y 50 premios para ofrecer a sus niños lectores en cada mes ya para la década de los cuarenta. Cabe señalar que dichos concursos y premiaciones no siempre eran organizados por Triquitraque, sino que participaron otras entidades culturales y económicas. Pues, se destaca el caso de la Sociedad ALA, la cual era una sociedad conformada por literatos que ofrecía premiaciones para dos ganadores de un concurso infantil en la ciudad de Heredia, el cual estaba siendo realizado en honor del poeta de dicha localidad Aquileo Echeverría, ofreciéndose una fiesta en dicho lugar el 4 de abril de 1937, celebrada en el extremo noreste del Parque Central, así fue inaugurado un monumento en honor de escritor, el cual consiste en una figura de mármol con el busto del mismo ${ }^{36}$.

\footnotetext{
34 Triquitraque: revista infantil, 1936-07-01: 16.

${ }^{35}$ Kysta Beam. Printing peace: cultural and pedagogical negotiation through children's periodicals in Costa Rica, 1912-1947 (Tesis para optar por el grado de Maestría de Artes en Estudios Latinoamericanos. USA: University of Illinois at Urbana-Champaign, 2016), p. 64.

${ }^{36}$ Triquitraque: revista infantil, 1937-05-01: 8.
} 
Empero, como se señaló en la recién sección anterior, se deja claro el hecho de que la revista para poder continuar y subsistir tuvo que buscar fondos de la $\mathrm{ANDE}^{37}$, ya que no pudieron sostener un proyecto de tal magnitud, solamente hasta que se dio la intervención de una institución pública. Además, de que otro factor relevante que se desprende de lo anterior fue la evidente importancia y popularidad que veían los educadores escolares en los números de la revista Triquitraque como material didáctico, el cual gestaron ajenamente por su continuidad con dicha asociación. Llama la atención el poder comprender por qué organizaciones de docentes como la ANDE e instituciones públicas del gran calibre de la Secretaría de Educación estaban tan interesados en la continuación del tiraje de Triquitraque, y como se ha dado a entender, la razón se encuentre en todos los materiales didácticos que lanzaba la revista, que iban más allá de las manualidades, siendo diversos formatos literarios los recursos educativos principales que utilizaba la revista para colocar las temáticas escolares actualizadas dependiendo del grado escolar que cursa el niño. Un caso que ejemplifica perfectamente esta idea anterior, se da con la literatura educativa que se presentaba como lecturas amenas para los primeros grados escolares que ofrecía la revista para el uso de los docentes, con el fin de aprender de gramática. De hecho, la codirección en conjunto de la revista expresó en el mes de marzo de 1940 una imagen de las maestras como agentes de cultura desde la difusión pedagógica de la revista Triquitraque.

Para continuar su consumo escolar, otra estrategia de financiamiento está en la alimentación de su compra por medio de las maestras, pues los coeditores utilizaron poemas, juegos, cuentos, preguntas y adivinanzas para el primer, segundo y tercer grado escolar, ya que tenían un fin para aprender a leer y divertirse; pero por otro lado, para el cuarto, quinto y sexto grado se utilizaron los mismos formatos literarios pero con el agregado de artículos con temáticas más complejas, como con el conocimiento de los avances tecnológicos por medio del gigantesco globo de gas Hindenburg ${ }^{38}$, siendo una temática más madura, pero siempre interactiva.

Otras veces se colocaba el material didáctico y otros suplementos escolares, pero sin ir dirigido a un grado respectivo, esto para mostrar temas generales como con la vida de los tiburones ${ }^{39}$. Así la revista desde el año 1937 se había declarado y presentado a sí misma de carácter didáctico para los escolares, más

37 Triquitraque: revista infantil, 1944-04-01: 2.

38 Triquitraque: revista infantil, 1937-06-01: 4-8.

39 Triquitraque: revista infantil, 1937-04-15: 6-9. 
allá de conocimientos generales ${ }^{40}$. El interés de la revista por ganarse la aprobación de los docentes escolares fue tanto que llegó a existir una pequeña sección continúa dirigida a los maestros para ofrecer la promoción de la revista como material didáctico ${ }^{41}$. En ese mismo número (44) a los pequeños lectores en la presentación de la revista a propósito del tema de la entrada de clases por medio del nombre "De nuevo a la escuela"42, revelaba el propósito del año según las maestras, pues se buscaba que los niños terminaran contando cuentos de Costa Rica, China, Japón y todos los países del mundo. Además, de que los niños deben de admirar a héroes patrios como Juan Santamaría ${ }^{43}$. Asimismo, aquel impreso representaba esa relación entre el incentivo escolar y Triquitraque como material didáctico, y un mercado comercial que apenas se estaba explorado con éxito, el de los impresos infantiles.

Otra estrategia de financiamiento más eficaz fue la publicidad, que se utilizó por medio de anuncios en las páginas de Triquitraque, la cual tenía fin crear un recurso mayor a las donaciones, las promociones y a las ventas del impreso. En otras palabras, el fin era publicitar tanto a sí misma como a entidades de todo tipo y sus productos ofrecidos en la revista Triquitraque, esto durante todo el periodo de duración de la revista, entre los años 1936 a 1947. Asimismo, se encontraron un total 186 entidades publicitadas (tanto estatales, privadas y la misma revista) con el fin de anunciar sus productos, servicios o promociones desde el primer año de publicación durante el periodo investigado ${ }^{44}$. Sin embargo, en esta sección no se centrará en ese lapso inicial, debido a que únicamente contiene autopublicidad y publicidad indirecta a la revista infantil misma, características que ya habían sido tratadas con anterioridad en el capítulo uno. Por ende, aunque para un mejor entendimiento se tomaron en cuenta la participación de los tipos de anuncios anteriores, lo importante será analizar lo ocurrido para el año 1937-1947. Además, hay que tener en cuenta que la revista publicitaba desde negocios propios hasta empresas transnacionales.

Aunque, algunos números de los últimos años tenían una publicidad en donde las entidades ajenas a la revista se observan de manera leve en aparición, pero siempre se lograba dar un debido espacio a la sección de anuncios y

\footnotetext{
40 Triquitraque: revista infantil, 1937-04-15: 2.

41 Triquitraque: revista infantil, 1937-05-01: 9.

42 Triquitraque: revista infantil, 1941-03-01: 2

43 Triquitraque: revista infantil, 1941-03-01: 2.

44 Ramírez Roldán José David, 2017. "Base de Datos: Triquitraque y su publicidad. 1936-1947”.
} 
anunciantes que se intercalaban entre los contenidos con normalidad ${ }^{45}$. Cabe señalar que, las mismas entidades sí se contaron en varias apariciones, pero no en cada una, para dejar de lado tomar en cuenta los anuncios repetitivos que hayan sido idénticos entre sí, y ver los que al menos hayan cambiado de tamaño, revelándose una aparición más o menos destacada.

Cuadro 1. 1

Número de entidades que publicaron anuncios en Triquitraque por provincia*.

\begin{tabular}{ccc}
\hline Provincia & Cantidad de anuncios & $\%$ \\
\hline San José & 129 & 86 \\
Alajuela & 5 & 3,4 \\
Heredia & 8 & 5,3 \\
Cartago & 8 & 5,3 \\
Guanacaste & 0 & \\
Puntarenas & 0 & \\
Limón & 0 & $\mathbf{1 0 0}$ \\
\hline total & $\mathbf{1 5 0}$ & \\
\hline
\end{tabular}

* No se tomaron en cuenta las entidades que no especificaron sus zonas de actividad como también sucede con el caso de las compañías internacionales.

Fuente: José David Ramírez Roldán, 2017. "Base de Datos: Triquitraque y su publicidad. 1936-1947."

Según los datos presentados en el cuadro anterior (1.1) de las 186 entidades encontradas solo 150 de ellas poseían una referencia geográfica (directa o indirecta) de su ubicación, pues hay 36 sobrantes que no se localizaron posiblemente debido a razones de peso, como que eran empresas internacionales (o industriales) o que simplemente no se refinanciaban. Asimismo, las cuatro provincias que conforman el Valle Central son las únicas zonas con aparición estelar, como también se podría afirmar que la gran mayoría se ubicaron en la provincia de San José, siendo un $86 \%$ del total de entidades tomadas en cuenta oriundas de dicha provincia, y en su mayoría específicamente de la ciudad de San José, como también por lo general sucedía con las ciudades cabeceras de las otras tres provincias vallecentralinas restantes, contando entre todas juntas apenas un $14 \%$ del total de entidades estudiadas. Este fenómeno revela la importancia de la revista en el Valle Central, y específicamente en la provincia y ciudad de San José para invertir en la publicidad del impreso, favoreciendo sus finanzas con empresas locales e internacionales.

45 Ibid. 


\section{Los autores de Triquitraque}

La revista Triquitraque se constituyó con contenidos literarios aptos para la lectura de los infantes y creados tanto autores nacionales como extranjeros, así que sus coeditores tendieron a publicar las obras de reconocidos escritores más relacionadas a consumirse como material didáctico y algunas veces con carácter lúdico.

Las publicaciones constantes buscaban generar en los jóvenes lectores conocimiento del contexto, tanto nacional como internacional, rescatándose en sus páginas los aportes culturales que hacía cada país del mundo a la literatura. Por ende, se publicaron obras de autores siempre y cuando se pudiera denotar en sus obras un contenido atractivo para la infancia costarricense, resaltándose con un lenguaje que fuera apropiado para los niños. Empero, en la primera sección de la presente parte se ofrece un análisis sistemático de los autores que colaboraron en los diversos formatos de los textos infantiles presentados en Triquitraque según su nacionalidad, después de esto, en los siguientes dos puntos restantes y considerando dichas colaboraciones de autores, se estudiarán a los mismos de origen extranjero con las variables de género. Sin duda alguna, la existencia de una revista infantil como Triquitraque durante los años treinta y cuarenta de la mano administrativa y directiva de destacados escritores tendría por lo menos, una relación evidente con el desarrollo de la cultura impresa de Costa Rica. Por ende, no solamente es posible estudiar cómo afectó este elemento a los autores nacionales en los diversos formatos literarios (como cuentos, poemas, leyendas, dramatizaciones, mitos y otros tipos de textos literarios o científicos en general) que brindaron sus colaboraciones o aparecieron con obras en las páginas de la revista, sino, que también ayudó a dar a conocer a los escritores de otros países, ya sean contemporáneos o de otros periodos históricos.

En el siguiente cuadro (2.1) se presentan únicamente dos variables en tres distintos tractos de años en que Triquitraque fue producida (1936 y 1939; 1940 y 1943; 1944 y 1947), según la condición de autores extranjeros y nacionales que participaron en los formatos más literarios, pero siendo atravesados con la variable de género. 
Cuadro 2. 1

Autores presentes dentro de la revista Triquitraque, 1936-1947

\begin{tabular}{lrrrrrrr}
\hline \multirow{2}{*}{ Año } & \multicolumn{2}{c}{ Nacionales } & \multicolumn{2}{c}{ Extranjeros } & \multicolumn{3}{c}{ Total } \\
& $\mathrm{M}$ & $\mathrm{F}$ & $\mathrm{M}$ & $\mathrm{F}$ & $\mathrm{M}$ & $\mathrm{F}$ & $\mathrm{T}$ \\
\cline { 2 - 8 } $1936-1939$ & 54 & 10 & 43 & 8 & 97 & 18 & 115 \\
$1940-1943$ & 11 & 1 & 13 & 4 & 24 & 5 & 29 \\
$1944-1947$ & 34 & 17 & 11 & 10 & 45 & 27 & 72 \\
\hline Total & 99 & 28 & 67 & 22 & 166 & 50 & $\mathbf{2 1 6}$ \\
\hline
\end{tabular}

Fuente: Ramírez Roldán José David, 2017. “Base de Datos: Triquitraque: contenidos generales. 1936$1947^{\prime \prime}$.

Este cuadro (2.1.) está conformado con datos sistematizados, siendo construido con el fin de revelar nombres repetidos de autores con cada obra de su autoría, ya que se toma en cuenta la aparición de los mismos en las distintas colaboraciones presentes en todos los números de la revista que estuvieran disponibles. Empero, la cantidad de años tomados en cuenta para cada tracto no son similares entre sí, pero tampoco diametralmente distintos. Esta diferencia es más evidente en el segundo tracto donde para el año 1943 no habría existencia alguna de números publicados por el impreso.

Entonces, el primer tracto es el más impactante en producción, pero a su vez se debe que se constituye más en años; el segundo tracto es el menos productivo, pero teniendo en cuenta la poca longevidad para construir el mismo; y el tercer tracto es más similar al primero en longevidad y consiguiente, en producción, ya que la revista pareciera ser constante en colaboraciones para los formatos literarios. Por ende, en el cuadro anterior (2.1.) se evidencia que entre los años 1936 y 1939 , los autores nacionales y extranjeros que colaboraron o aparecieron con sus trabajos corresponden a un total de 115 apariciones de diversas obras hechas tanto por hombre como por mujeres, que a su vez es constituido por un total en términos de la variable de género con un valor en el sector masculino de 97 participaciones y el femenino únicamente con 18.

En término específicos, para el primer tracto la cantidad en términos de extranjeros serían 43 en el sector masculino y 8 en el femenino. Y para el segundo periodo se evidencia como es el único momento en que los autores extranjeros (contándose hombre y mujeres) superan a los nacionales en cantidad, ya que a nivel internacional en ese contexto había 13 hombres y 4 
mujeres, concluyendo a nivel total por género con 24 hombres y cinco mujeres, dejando un total general entre los dos géneros de 29 participaciones, lo anterior es contando tanto a los nacionales como a los extranjeros. Por su lado, el tercer tracto dejó en evidencia nuevamente una mayor participación masculina que rondaba a nivel nacional con 34 apariciones, y a nivel femenino con 17. A nivel internacional las cifras en este tercer periodo son más parejas, con una aparición de 11 hombres y 10 mujeres; dejando un total de 45 hombres y 27 mujeres, siendo el contexto con mayor participación femenina, para un total de 72 colaboraciones entre los dos géneros.

En resumen, en todos los tres periodos, los hombres participaron más de tres veces que las mujeres, además, se podría concluir que hay una mayor participación (o aparición) femenina de origen nacional en comparación con el ámbito internacional. Lo anterior, posiblemente, es debido a que el arte de la escritura tanto en el plano nacional como internacional desde la tradición folklórica que era lo que más rescataba la revista ha estado a través de la historia humana en manos del género masculino por el acceso más amplio a la educación y no corresponde tanto a un proceso de feminización de la educación.

Algo interesante sucedió con la tradición francesa (John James Audubon, Charles-Louis Philippe, Gabriel Meurier y Jean de La Fontaine) como con la alemana (hermanos Grimm), ya que la revista por medio de arreglos o versiones propias se apropiaron de obras y personajes populares de la tradición oral europea, como sucedió con el personaje ficticio de Caperucita Roja, donde el impreso hizo una versión alternativa donde el lobo no se comió a Caperucita ${ }^{46}$. Otro dato específico interesante de los autores extranjeros para poder comprender la explotación de escritores difuntos como Robert Browning ${ }^{47}$, Alfred Tennyson ${ }^{48}$, Frances Browne ${ }^{49}$, Emma Pérez ${ }^{50}$, Rafael Olivares Figueroa 51 y Giovanni Pascoli52, por citar algunos cuantos, ya que las obras clásicas infantiles de origen extranjero fueron apropiadas por traducciones al español hechas por la misma revista o sus coeditores, como sucedió con una obra de Loure Smith ${ }^{53}$ arreglada con su respectiva traducción por Carlos Luis Sáenz.

\footnotetext{
46 Triquitraque: revista infantil, 1940-07-01:3

47 Triquitraque: revista infantil, 1936-05-15: 5-6.

48 Triquitraque: revista infantil, 1937-05-01, 17.

49 Triquitraque: revista infantil, 1941-08-01: 4-7.

50 Triquitraque: revista infantil, 1937-06-01: 14.

51 Triquitraque: revista infantil, 1937-06-01:14.

52 Triquitraque: revista infantil, 1941-05-01: 2.

53 Triquitraque: revista infantil, 1936-08-01: 4-5.
} 
Pareciera que se buscó más la participación activa de autores y autoras contemporáneas en un impreso infantil como Triquitraque, reluciendo figuras como: Ernesto Mario Barreda ${ }^{54}$, Adriano Del Valle 55 , Salvador Rueda ${ }^{56}$, Jaime

Torres Bodet ${ }^{57}$, Eleazar Huertas ${ }^{58}$, Ildefonso Pereda Valdés ${ }^{59}$, Franco Luis Leopoldo ${ }^{60}$, Rafael Alberti Merello ${ }^{61}$ y Jorge Carrera Andrade ${ }^{62}$, entre otros. De dicha lista de nombres extranjeros lo más interesante de observar es que son literatos del idioma español (castellano), evidenciado que la revista fue más un espacio de difusión de temas contemporáneos y cosmopolitas, por ende, no se recurrió a realizar arreglos de obras de autores latinoamericanos o españoles, como si sucedió con el caso de la literatura infantil nacional y clásica.

A un lado a lo anteriormente dicho, también hubo participantes costarricenses que eran infantes en su momento, sin distinción de género hubo colaboración de niños y niñas, ya que el caso más interesante es el de María Eugenia Dengo Obregón de la Escuela Rafael Moya de Heredia, quien había ganado el primer premio en el concurso abierto para dar nombra a la revista en mes de marzo de 1936, el dato que más llama la atención es que esta misma infanta fue ganadora del premio Aquileo Echeverría durante su adultez. En aquel número $\left(1^{\circ}\right)$ del impreso se puede apreciar como la niña Dengo Obregón expresaba con sus propias palabras que quería que "...la revista de los niños se llame Triquitraque, para que meta mucha bulla y todos gocemos mucho cuando la leamos"63. La mención anterior simplemente revela el papel central que la revista le otorgó a la infancia, dándoles voz a esta población como posibles autores nacionales, tanto para hombres como mujeres.

\section{Temáticas y contenidos de Triquitraque}

En esta parte se estudia cómo se presentaban los diversos contenidos literarios de la revista Triquitraque para entender cómo se dio el desarrollo didáctico del

\footnotetext{
54 Triquitraque: revista infantil, 1937-09-01: 7.

55 Triquitraque: revista infantil, 1937-07-01: 10.

56 Triquitraque: revista infantil, 1937-07-01: 11.

57 Triquitraque: revista infantil, 1947-06-01: 3.

58 Triquitraque: revista infantil, 1937-07-01: 10.

59 Triquitraque: revista infantil, 1936-06-01:12.

60 Triquitraque: revista infantil, 1938-06-01: 9.

61 Triquitraque: revista infantil, 1936-09-01: 10.

62 Triquitraque: revista infantil, 1936-09-01: 10.

63 Triquitraque: revista infantil, 1936-05-15: 4.
} 
impreso. En el siguiente cuadro (3.1.) se apreciarán los tipos de texto literarios que fueron recopilados sistemáticamente en la revista Triquitraque por medio del formato de poema, cuento, leyenda, dramatización, texto histórico, texto sobre salud y otros tipos de textos literarios. Lo anterior, es presentado en tres tractos de años (periodos): el primero entre 1936 y 1939, el segundo entre 1940 y 1943, y el tercero de 1944 a 1947.

Cuadro 3. 1

Contenido literario de la revista según su formato, 1936-1947

\begin{tabular}{lrrrrrrrr}
\hline Fecha & Poema & Cuento Leyenda & Dramatización & $\begin{array}{r}\text { Texto } \\
\text { histórico }\end{array}$ & $\begin{array}{c}\text { Texto } \\
\text { sobre } \\
\text { salud }\end{array}$ & Otros & Total \\
\hline $1936-1939$ & 71 & 60 & 1 & 9 & 27 & 2 & 111 & 281 \\
$1940-1943$ & 34 & 43 & 4 & 3 & 23 & 1 & 53 & 161 \\
$1944-1947$ & 33 & 51 & 4 & 7 & 27 & 15 & 75 & 212 \\
\hline Total & 138 & 154 & 9 & 19 & 77 & 18 & 239 & $\mathbf{6 5 4}$ \\
\hline
\end{tabular}

Fuente: Ramírez Roldán José David, 2017. “Base de Datos: Triquitraque: contenidos generales. 1936$1947^{\prime \prime}$.

En el cuadro anterior (3.1.), se aprecia como todos los formatos tomados en cuenta (exceptuando a la sección de "otros") fueron empleados por la revista Triquitraque durante toda su publicación, siendo los dos principales recursos más utilizados los cuentos (154 apariciones) y los poemas (138 apariciones), esto según los totales de todos los tres periodos tomado en cuenta. El papel del texto histórico es el tercer recurso con mayor aparición (77 veces), seguido por las dramatizaciones (19 veces), los textos de salud (18 veces) y las leyendas (9 veces). Cabe señalar, que el cuadro (3.1.) tiene una categoría denominada "otros", la cual es la más recurrida en todos los tractos anuales y, por ende, en apariciones totales, con un total de 229 veces en todos los años disponibles. Esto debido a que está conformada por artículos científicos, adivinanzas, juegos, trabalenguas, mapas, charadas, espacios políticos contra el fascismo, relatos, estampas, trabajos de infantes, preguntas, mitos, solicitudes de corresponsales, anécdotas, excursiones, cuadrígramas, pensamientos, formalismos, lecturas escolares, fábulas, horóscopos, pasatiempos, crucigramas, cartas, experimentos, chistes, adivinanzas, recetas de cocina, ejercicios mentales, calendarios meteorológicos y material didáctico ${ }^{64}$. Lo anterior, revela la riqueza de recursos de la revista, concluyendo con la inmensa suma de seiscientos

${ }^{64}$ Ramírez Roldán José David, 2017. “Base de Datos: Triquitraque: contenidos generales. 1936-1947”. 
cincuenta y cuatro apariciones de diversos formatos literarios e interactivos que se empleaban en la otra como total final.

En los números de la revista Triquitraque es apreciable que los contenidos de lo que se ha colocado en la sección de "otros" en su mayoría son recursos difíciles de encanillar o con muy pocas apariciones como formatos literarios específicos, sin embargo, algunos son extensos. Esto solo deja en evidencia el conocimiento enciclopédico de la literatura y el folklor, tanto nacional como internacional, entendido por los productores de la revista, en donde se rescató fabulas rebuscadas de la tradición malgache ${ }^{65}$ (una lengua malayo-polinesia) hasta conocidos mitos griegos como el de Filemón y Baucis ${ }^{66}$. Aunque la mayor parte del tiempo eran lecturas escolares, adivinanzas, crucigramas y artículos informativos de temas variados, científicos la mayoría de las veces ${ }^{67}$. Sin embargo, los espacios políticos contra el fascismo tenían un amplio espacio en las páginas de la revista y eran producidos por la misma dirección y administración del impreso. Por consiguiente, Asimismo, en las páginas de Triquitraque es apreciable como se desarrolló a mitad del período de publicación una evidente politización directamente relacionado con la Segunda Guerra Mundial (1939 y 1945).

Lo anterior, se expresa mejor en el año de 1942 (y en otros constantes números hasta el fin del conflicto bélico internacional) en donde se les explica a los niños sobre el Frente Nacional Anti-Nazi, y la propuesta antifascista que ofrece la codirección de la revista por medio del denominado Club de la $\mathrm{V}$ (de la victoria), una organización para los niños que llamaba a la democracia y a luchar contra la proliferación de ideas nacionalsocialistas o fascistas durante dicho conflicto bélico ${ }^{68}$.

Los textos históricos parecieran estar siempre calculados en apariciones, pues era un recurso bastante explotado por la misma dirección de la revista. Su alto uso en algunas ocasiones tenía relación con la producción de otros impresos como los eran San Selerín 69 (extinta revista costarricense infantil para el momento) y La Tribuna ${ }^{70}$ (un reconocido periódico nacional). Lo más llamativo de esta sección es que únicamente solo hubo textos producidos por la misma

\footnotetext{
65 Triquitraque: revista infantil, 1939-03-01: 12.

66 Triquitraque: revista infantil, 1937-05-01: 4-6.

67 Ramírez Roldán José David, 2017. "Base de Datos: Triquitraque: contenidos generales. 1936-1947".

${ }^{68}$ Triquitraque: revista infantil, 1942-05-01: 14-15.

69 Triquitraque: revista infantil, 1940-08-01: 10-11

70 Triquitraque: revista infantil, 1944-06-01: 10-12,
} 
revista, estudiosos costarricenses y americanos en general, yendo desde historias de tradición oral estadounidense como el héroe del trigo norteamericano llamado Marco Alfredo Carleton ${ }^{71}$ hasta textos producidos por la independentista guatemalteca María Dolores Bedoya ${ }^{72}$, además de temas tan variados y generales como los materiales arqueológicos de la biblia ${ }^{73}$. Por ende, no hay textos históricos sobre temáticas relacionadas a tradiciones europeas como tal, sino sobre la historia de la humanidad en general o pertenecientes al continente americano o relacionadas al mismo. Otro factor que amplio la participación del formato del texto histórico fue el uso de las biografías, al rescatarse figuras históricas de la política como el ex presidente decimonónico de Costa Rica Jesús Jiménez Zamora74 (de 1863 a 1866 y 1868 a 1870) y también a Cleto González Víquez ${ }^{75}$ (de 1906 a 1910 y 1928 a 1932), sin dejar de lado la figura histórica del Márquez de Talamanca ${ }^{76}$. Además, se rescataron figuras nacionales e internacionales de corte intelectual como Aquileo Echeverría77, Joaquín García Monge ${ }^{78}$, Clodomiro Picado ${ }^{79}$, Anastasio Alfaro ${ }^{80}$, Magón ${ }^{81}$ y el cubano José Martí82. Empero, la constante aparición de esta categoría de textos históricos se debe a la existencia de celebraciones patrias como el 15 de setiembre (día de la independencia centroamericana)83, 12 de octubre (descubrimiento de América) ${ }^{84}$ y 25 de julio (anexión del Partido de Nicoya) ${ }^{85}$.

Los textos sobre salud no tienen la misma importancia en apariciones según el cuadro (3.1.), sin embargo, pasan de aparecer únicamente una o dos veces entre 1936 y 1943 hasta llegar en el tercer tracto a la cifra de 15 apariciones, evidenciando la importancia que empezó adquirir dicha sección. Esto es debido a que después del mes de abril de año 1944 se empieza a colocar artículos a modo de anuncios creados por la Jefatura de Sanidad de San José en ese mismo año86, llamando a los maestros y alumnas a no botar basura en las calles capitalinas. Por ende, Triquitraque como impreso no solo llamaba la atención de

\footnotetext{
71 Triquitraque: revista infantil, 1941-07-01: 10-11, 15-16.

72 Ramírez Roldán José David, 2017. "Base de Datos: Triquitraque: contenidos generales. 1936-1947".

73 Triquitraque: revista infantil, 1939-05-01: 11-14.

74 Triquitraque: revista infantil, 1939-11-01: 10.

75 Triquitraque: revista infantil, 1940-05-01: 3-4.

76 Triquitraque: revista infantil, 1947-07-01: 4

77 Triquitraque: revista infantil, 1947-09-01: 3-4.

78 Triquitraque: revista infantil, 1944-09-01: 13-14.

79 Triquitraque: revista infantil, 1947-05-01: 2-4.

80 Triquitraque: revista infantil, 1946-04-01: 9.

81 Triquitraque: revista infantil, 1936-09-01: 5-6.

82 Triquitraque: revista infantil, 1936-09-01: 4.

83 Triquitraque: revista infantil, 1947-09-01: 8-10.

${ }^{84}$ Triquitraque: revista infantil, 1941-05-01: 6.

85 Triquitraque: revista infantil, 1940-08-01: 10-11.

86 Triquitraque: revista infantil, 1944-06-01: 16.
} 
intelectuales de la literatura infantil, sino que también de personas de otros campos como la medicina, como en el caso del Dr. Manuel Gonzáles Rivera, quien en esta misma sección educó a los niños con cuentos infantiles a propósito de la higiene personal ${ }^{87}$. Pero también se tocaban otros temas como el contacto con el dinero y el cuidado higiénico con el mismo ${ }^{88}$. Además, para los últimos años del impreso transcurridos dentro del tercer tracto anual, casi siempre en las páginas finales de la revista, el Servicio Cooperativo Inter-Americano de Salud Pública junto con el Departamento de Educación Sanitaria empezó a crear artículos informativos ${ }^{89}$, los cuales estaban bastante mezclados con formatos como cuentos, prosa, leyendas y adivinanzas, recursos que ya eran conocidos por los lectores recurrentes del impreso.

\section{Conclusiones}

Triquitraque fue un órgano originalmente administrado y dirigido por maestras de maternal y posteriormente por militantes comunistas que a su vez también era reconocidos intelectuales en el campo de la literatura infantil, convirtiéndola no solamente en una revista de cultura general para niños, sino, que también con fines educativos. Así, la revista infantil Triquitraque rescató los aportes culturales dados por las figuras históricas tanto nacionales como extranjeras, empero, y solo es posible encontrar en sus páginas obras con temáticas literarias en un lenguaje infantil, dejándose la política de lado, aunque sus productores estaban dentro de una fuerte actividad política. Sin embargo, la revista sí reveló sus intenciones antifascistas, creando hasta brigadas infantiles para luchar contra el nazismo y el fascismo, pero entendiéndose en un contexto bélico durante la Segunda Guerra Mundial y los años cercanos a la última Guerra Civil de Costa Rica.

Por consiguiente, queda claro que Triquitraque estaba inmerso en un contexto difícil para la continuación de una revista infantil pero que logró hacerse fama con el tiempo. Empero, su existencia no fue únicamente para dar una ganancia económica para su debida continuación y lograr lucrarse, sino tenía el fin de popularizar sus contenidos en el ámbito cultural costarricense hasta llegar a entrar en la esfera educativa por medio de la protección de instituciones educativas de la época, como la Ande y la misma Secretaría de Educación. Así,

87 Triquitraque: revista infantil, 1946-04-01: 13-15.

88 Triquitraque: revista infantil, 1946-07-01: 14-15.

89 Triquitraque: revista infantil, 1946-05-01: 14-15. 
es apreciable como la revista utilizó una imagen de contenido lúdico-escolar que terminó mezclándose con el apoyo de organizaciones de docentes y hasta institucional del mismo estado costarricense para su debido financiamiento, tanto que la revista prácticamente venía siendo en los años cuarenta un suplemento escolar más, y posiblemente el más atractivo y popular de la infancia en aquellos años, esto concluido gracias a su alto número de consumidores a nivel nacional, como la cifra de 10.000 lectores. Finalmente, se podría inferir que, aunque Triquitraque no fue un órgano como tal de la política editorial del Partido Comunista de Costa Rica, sí se puede afirmar que la revista tenía conexiones culturales y pedagógicas con diversos intelectuales en todo tipo de campos del conocimiento humano gracias a sus coeditores. Así, sus coeditores comunistas tuvieron siempre una estratégica cautela en los contenidos presentado para que no se les relacionara directamente con el Partido Comunista, por ende, ofrecieron todas las condiciones favorables en la producción del impreso. 


\section{FUENTES}

Ramírez, J. D. (2017). Triquitraque, revista infantil (1936-1947). Trabajo de graduación en Bachiller de Historia. Universidad de Costa Rica. San José, 2017.

Ramírez, J. D. (2017). Base de Datos: Triquitraque y su publicidad. 1936-1947.

Ramírez, J. D. (2017). Base de Datos: Triquitraque: contenidos generales.19361947.

Asociación de Maestras de Kindergarten (Carlos Sáenz, Luisa González, Lilia Ramos y Adela Ferreto). Triquitraque: revista infantil, 1939-1946.

\section{BIBLIOGRAFÍA}

Beam, K. (2016) Printing peace: cultural and pedagogical negotiation through children's periodicals in Costa Rica, 1912-1947. Tesis para optar por el grado de Master of Arts in Latin American Studies. USA: University of Illinois at UrbanaChampaign, Latin American and Caribbean Studies Program, Latin American Studies.

Botey, A. M. (2005a). Las representaciones sociales de la pobreza en la Costa Rica de la década de 1930. Viales, R. J. (comp.). Pobreza e historia en Costa Rica: determinantes estructurales y representaciones sociales del siglo XVII a 1950. San José: EUCR: pp. 273-294.

Botey, A. M. (2005b). Costa Rica entre guerras: 1914-1940. San José: EUCR.

Botey, A. M. (1981). La fundación del partido comunista de Costa Rica, 1931. Tesis para optar por el grado de licenciatura en historia. San José: Universidad de Costa Rica, Facultad de Ciencias Sociales, Escuela de Historia.

Contreras, G. (2009). Arnoldo Ferreto Segura y el Partido Comunista de Costa Rica en la lucha por la segunda y auténtica Independencia Nacional. Revista Estudios 22, 65-84.

Dobles, M. (1981) Literatura infantil. San José: EUNED. 
Molina, I. \& Lehoucq. F. E. (1999). Urnas de lo inesperado. Fraude electoral y lucha política en Costa Rica (1901-1948). San José: EUCR.

Molina, I. (2010). Los comunistas y la publicidad en Costa Rica. El caso del periódico Trabajo (1937-1948). Secuencia. Revista de Historia y Ciencias Sociales 77, 61-87.

Molina, I. (2007). Ricardo Jiménez y los comunistas en la Costa Rica de la década de 1930. Inter.c.a.mbio 4 (5), 71-100.

Rodríguez, A. P. (2009) Dividing the Isthmus: Central American Transnational Histories, Literatures, and Cultures. USA: University of Texas Press.

Zeledón, E. (2003). Biografías de costarricenses. Heredia: EUNA. 